\title{
Conseqüências em Longo Prazo da Deficiência do Hormônio de Crescimento
}

\begin{abstract}
RESUMO
Este artigo descreve as conseqüências puras, em longo prazo, da deficiência isolada e vitalícia do hormônio de crescimento $(\mathrm{GH})$ porque usa um modelo único de resistência ao hormônio liberador do $\mathrm{GH}$ (GHRH), em virtude da mutação homozigótica no gene do receptor do $\mathrm{GHRH}$, em uma centena de indivíduos acometidos. Elas incluem baixa estatura grave com estatura final entre -9,6 a -5,2 desvios-padrão abaixo da média, com redução proporcional das dimensões ósseas, redução do volume da adenohipófise corrigido para o volume craniano e da tireóide, do útero, do baço e da massa ventricular esquerda, todos corrigidos para a superfície corporal, em contraste com o tamanho de pâncreas e fígado, maior que o de controles, quando igualmente corrigidos. As alterações características da composição corporal incluem redução acentuada da quantidade de massa magra $(\mathrm{kg})$ e aumento do percentual de gordura com depósito predominante no abdome. Nos aspectos metabólicos são encontrados aumento de colesterol total e LDL, redução de insulina e do índice de resistência à insulina homeostasis model assessment, acompanhados de aumento da proteína $C$ reativa de alta sensibilidade e da elevação da pressão arterial sistólica nos adultos, embora sem evidências de aterosclerose precoce. Outros achados incluem resistência óssea menor, embora acima do limiar de fraturas, puberdade atrasada, fertilidade normal, paridade diminuída, climatério antecipado e qualidade de vida normal. (Arq Bras Endocrinol Metab 2008; 52/5:745-749)
\end{abstract}

Descritores: Deficiência de hormônio de crescimento; Hormônio do crescimento.

\begin{abstract}
Long Time Consequences of the Growth Hormone Deficiency.

This article describes the long time consequences of the isolated and lifetime growth hormone $(\mathrm{GH})$ deficiency using a single model of $\mathrm{GH}$ releasing hormone resistance (GHRH) due to a homozygous mutation in the GHRH receptor gene, in a hundred of subjects. These consequences include severe short stature with final height between -9.6 and $\mathbf{- 5 . 2}$ standard deviations below of the mean, with proportional reductions of the bone dimensions; reduction of the anterior pituitary corrected to cranial volume and the thyroid, the uterus, the spleen and left ventricular mass volume, all corrected to body surface, in contrast of pancreas and liver size, bigger than in controls, when equally corrected. Body composition features included marked reduction in the amount of fat free mass $(\mathrm{kg})$ and increase of fat mass percentage, with predominant abdominal deposit. In the metabolic aspects, we find increase in the total cholesterol and LDL cholesterol; reduction of the insulin and the insulin resistance assessed by Homeostasis model assessment; increase of ultra sensitive $C$ reactive protein and systolic body pressure in adults, although without evidences of premature atherosclerosis. Other findings include smaller bone resistance, although above of the threshold of fractures, delayed puberty, normal fertility, small parity, anticipated climacteric and normal quality of life. (Arq Bras Endocrinol Metab 2008; 52/5:745-749)
\end{abstract}

Keywords: Growth hormone deficiency; Growth hormone. revisão

\author{
Carla R. P. Oliveira
}

Rossana M. C. Pereira

José A. S. BARRETO-FILHO

Manuel H. Aguiar-Oliveira

Serviço de Endocrinologia do Hospital Universitário da

Universidade Federal de Sergipe, Aracaju, SE, Brasil.
Recebido em 23/5/2008

Aceito em 30/5/2008 


\section{INTRODUÇÃO}

$\mathrm{E}$ VANS E LONG, em 1921, (1) demonstraram a existência do hormônio de crescimento $(\mathrm{GH})$ e, duas décadas após, Li e Evans isolaram e sintetizaram o hormônio cristalino (2). O GH tem importantes funções no organismo, além do papel que lhe sugere o nome (3). Neste trabalho será analisado o papel do GH sobre o crescimento, a composição corporal, os metabolismos lipídico, glicídico e ósseo, o risco cardiovascular, a vida reprodutiva e a qualidade de vida. Para tanto, foi utilizado como modelo um experimento da natureza, em que 105 indivíduos de uma mesma família apresentam deficiência genética isolada do GH de herança autossômica recessiva, com níveis extremamente baixos de GH (deficiência de GH tipo IB) (4), por causa da mutação homozigótica IVSI +1 G $\rightarrow$ A no gene do receptor do hormônio liberador do GH (GHRH-R), descrita em Itabaianinha, Sergipe $(5,6)$. Embora as conseqüências da deficiência do $\mathrm{GH}$ (DGH) possam diferir quando isolada ou associada a outros hormônios hipofisários, quando iniciada na infância ou na idade adulta, quando grave ou moderada, o modelo em questão é único para se estudar as conseqüências puras da deficiência isolada do GH (DIGH) vitalícia e não-tratada no organismo humano. Esta singularidade será apresentada no presente trabalho, deixando para os leitores interessados as controvérsias sobre os outros modelos, diferentes do a seguir apresentado.

\section{CRESCIMENTO SOMÁTICO}

A DIGH de Itabaianinha provoca acentuada baixa estatura de início pós-natal com estatura final entre 107 e $136 \mathrm{~cm}$ ou $-5,2$ a $-9,6$ desvios-padrões abaixo da média estatural. Esta baixa estatura é proporcional, no que diz respeito aos seguimentos corporais, haja vista que o crescimento do tecido ósseo é o responsável pelo estabelecimento da altura final (7). Dessa forma, mãos, pés, membros, tórax e crânio são proporcionalmente reduzidos, embora os dentes sejam de dimensões normais. É importante ressaltar que as dimensões de algumas estruturas, como o volume da adenohipófise corrigido para o volume craniano (8), volume da tireóide (9) e massa ventricular esquerda corrigidos para a superfície corporal (10), são menores que nos controles normais. A hipoplasia da adenohipófise provavelmente decorre da não-proliferação dos somatotrofos devidos à resistência ao GHRH, e é um aspecto muito importante embora não universal nas mutações que envolvam o receptor do GHRH (8,11-18). Já para o estabelecimento do volume da tireóide e da massa ventricular esquerda são críticos a atividade do $\mathrm{GH}$ e seu principal mediador, o fator de crescimento semelhante à insulina tipo I ou IGF-1. Este fenômeno de redução preferencial de alguns órgãos é visto na ultra-sonografia dos órgãos intra-abdominais, na qual útero e baço apresentam redução das dimensões corrigidas para a superfície corporal; próstata e ovários são equivalentes aos controles normais; e rins, pâncreas e fígado são maiores quando corrigidos para a superfície corporal (19).

\section{COMPOSIÇÃO CORPORAL}

Um aspecto fundamental neste modelo de DIGH genética e grave é a alteração da composição corporal, com redução importante da massa magra desde a infância, com agravamento na puberdade $(20,21)$ e estabelecendo nos adultos $(10,22)$ um padrão de quantidade de massa magra $(\mathrm{kg})$ bastante reduzida com o percentual de gordura (\%) aumentado, não a massa da gordura $(\mathrm{kg})$. Outro dado importante é um predomínio da gordura abdominal observada em todas as faixas etárias.

\section{METABOLISMO LIPÍDICO}

As crianças e os adolescentes com DIGH de Itabaianinha apresentam níveis elevados de colesterol LDL e de colesterol total em comparação com os controles da mesma região $(21,23)$, que continuam durante toda a vida $(10,22)$, efeito eventualmente associado à redução da expressão dos receptores hepáticos para o colesterol LDL. Estudos em ratos e humanos mostraram efeito importante de GH no metabolismo hepático do colesterol. A administração de GH estimula a expressão dos receptores hepáticos do colesterol LDL, aumentando o catabolismo de LDL-C pelo fígado (24). O aumento de colesterol plasmático com a idade (25) ou o hipotireoidismo é pelo menos parcialmente secundário à diminuição da atividade nos receptores do colesterol LDL induzida pela diminuição na secreção de GH (26). Não há redução nos níveis de colesterol HDL, nem diferenças nos níveis de triglicérides entre os indivíduos com DIGH de Itabaianinha e os controles da mesma região. 


\section{METABOLISMO GLICÍDICO}

Embora a DIGH moderada de início na idade adulta seja associada à insulino-resistência $(27,28)$, os indivíduos com DIGH de Itabaianinha apresentam níveis insulínicos reduzidos e dos valores de índice de resistência à insulina homeostasis model assessment, HOMA-IR (glicemia $(\mathrm{mMol}) \times$ insulina $(\mathrm{uU} / \mathrm{mL}) \div 22,5)$ menores que dos controles da mesma região $(10,22)$, até em idade avançada. Estudos de sensibilidade à insulina utilizando teste endovenoso de tolerância à glicose, com a aplicação da análise do modelo mínimo das variações das concentrações de glicose e de insulina após a administração da glicose (29), deverão ser realizados nestes indivíduos para melhor avaliar a secreção insulínica.

\section{METABOLISMO ÓSSEO}

A DGH, de maneira geral, está associada com menor densidade mineral óssea e eventual risco de fraturas $(30,31)$. Os indivíduos com DIGH de Itabaianinha apresentam redução da resistência óssea, medida pela ultra-sonografia do calcâneo, embora acima do limiar de fraturas, correspondendo ao relato da baixa freqüência de fraturas neles (32).

\section{RISCO CARDIOVASCULAR}

Conforme assinalado, os indivíduos com esta forma de DIGH apresentam menor massa magra, maior percentual de gordura, distribuição central de gordura, aumento do colesterol total e, na idade adulta, pressão arterial sistólica mais elevada, embora com índice de massa ventricular esquerda menor (10). Com tantos fatores de risco cardiovascular associados, seria esperado que estes indivíduos apresentassem aterosclerose prematura, o que não foi evidenciado seja pela ecocardiografia de estresse ou pela aferição da espessura médio-intimal da carótida, ainda que os níveis de proteína $\mathrm{C}$ reativa de alta sensibilidade, um marcador inflamatório de risco cardiovascular, sejam marcadamente elevados (22). Observou-se que a DIGH genética e grave é menos deletéria em termos de aterosclerose que a DIGH adquirida ou moderada, talvez pela dificuldade de proliferação das células musculares lisas, etapa primordial para o crescimento de placa aterosclerótica, quando o eixo GH/IGF-l é vitalício e gravemente deprimido. Por outro lado, a redução menos grave do
IGF-1 e de início na idade adulta pode levar à apoptose daquelas células, provocando a rotura da placa e os eventos cardiovasculares na DIGH adquirida ou moderada $(22,33,34)$.

\section{VIDA REPRODUTIVA}

Os indivíduos com DIGH de Itabaianinha apresentam a puberdade moderadamente atrasada (23) e o climatério de início mais precoce (35), embora este seja essencialmente normal. Os indivíduos de ambos os sexos são férteis, embora a paridade das mulheres com DIGH seja menor que a das mulheres da região. Esta paridade pode se dever ao medo da cirurgia cesariana, pouco disponível no passado ou à menor duração da vida reprodutiva (35).

\section{QUALIDADE DE VIDA (QV)}

Embora numerosas citações refiram piora da QV em indivíduos com DGH, a maioria é em DIGH adquirida e de início na idade adulta $(36,37)$. Novamente é peculiar que o índice total de QV avaliado pelo questionnaire of life satisfaction (36), questionário específico e validado para a DIGH, foi igual ao dos controles da mesma região e superiores ao de todas as séries de DIGH, analisados na Europa e nos Estados Unidos (36). Dessa forma, a DIGH genética e grave em uma população do nordeste brasileiro em processo de urbanização não parece comprometer a QV (38).

\section{CONSIDERAÇÕES FINAIS}

Nesse modelo de DIGH genético e grave há repercussões evidentes sobre o crescimento, a estatura final e os diversos órgãos. Alia-se a isso complexo conjunto de alterações metabólicas e de composição corporal com fatores adversos ou protetores para a saúde em geral e sem piora da QV. O tratamento com GH subcutâneo diário em crianças, além de provocar o ganho estatural, melhora o perfil lipídico e a composição corporal $(21,23)$. Resultados semelhantes sobre o perfil lipídico e a composição corporal foram obtidos em adultos, sem repercussões sobre a glicemia ou a hemoglobina glicada, com a administração do GH de depósito subcutâneo a cada 15 dias (34), acrescido de melhora da remodelação óssea, de padrão anabólico, com conseqüente aumento da resistência óssea (32). 
Um possível efeito adverso deste tratamento foi a eventual aceleração da aterosclerose (34), limitada pela suspensão do tratamento. Assim, a terapêutica em GH no adulto deve levar em conta o equilíbrio entre os fatores positivos e os fatores adversos. Embora a generalização destes achados para as formas de DIGH adquiridas e menos graves encontradas na clínica não deva ser assegurada, uma menção de cuidado deve ser assinalada por ocasião de usos não completamente estabelecidos do GH, especialmente em indivíduos adultos.

\section{REFERÊNCIAS}

1. Evans HM, Long JA. The effect of the anterior lobe administered intraperitoneal upon growth maturity an oestrus cycles of the rat. Anat Rec. 1999;21:62-3.

2. Li CH, Evans HM. Chemistry of anterior pituitary hormones. In: Pincus G, Thieman KV, editors. The hormones. New York: New York Academic Press; 1948.

3. Souza AHO, Salvatori R, Martinelli Jr CE, Carvalho WMO, Menezes CA, Barretto ESA, et al. Hormônio do crescimento ou somatotrófico: novas perspectivas na deficiência isolada de $\mathrm{GH}$ a partir da descrição da mutação no gene do receptor do GHRH nos indivíduos da cidade de Itabaianinha, Brasil. Arq Bras Endocrinol Metab. 2004;48:406-13.

4. Nishi Y, Masuda H, Nishimura S, Kihara M, Suwa S, Tachibana $\mathrm{K}$, et al. Isolated human growth hormone deficiency due to a hGH gene deletion with (TYPE IA) and without (the Israelitype) hGH antibody formation during hGH therapy. Yokoama - Japão. Acta Endocrinology (Copenh). 1990;122:267-71.

5. Salvatori $\mathrm{R}$, Hayashida $\mathrm{CY}$, Aguiar-Oliveira $\mathrm{MH}$, Phillips III JA, Souza AHO, Gondo RG, et al. Familial isolated growth hormone deficiency due to a novel mutation in the growth hormone releasing hormone receptor. J Clin Endocrinol Metab. 1999;84:917-23.

6. Aguiar-Oliveira MH, Gill MS, Barretto ESA, Alcântara MRS, Miraki-Moud F, Menezes CA, et al. Effect of growth hormone $(\mathrm{GH})$ deficiency due to a mutation in the $\mathrm{GH}$-releasing hormone receptor on insulin-like growth factors (IGFs), IGF-binding proteins, and ternary complex formation throughout life. J Clin Endocrinol Metab. 1999;84:4118-26.

7. Tanner JM. Normal growth and techniques of growth assessment. Clinics in Endocrinology and Metabolism. 1986;15:411-51.

8. Oliveira HA, Salvatori R, Kraus, Oliveira CRP, Silva PCR, AguiarOliveira $\mathrm{MH}$. Magnetic ressonance imaging study of pituitary morphology in subject6s homlozigous and heterozigous for a null mutation of the GHRH receptor gene. Eur J Endocrinol. 2003;148:427-32

9. Alcântara MRS, Salvatori R, Alcântara PRS, Nóbrega LMA Campos VS, Oliveira ECO, et al. Thyroid morphology and function in adults with untreated isolated growth hormone deficiency. Journal of Clinical Endocrinology and Metabolism. 2006;91:860-4.

10. Barreto-Filho JAS, Alcântara MRS, Salvatori R, Barreto MA, Sousa ACS, Bastos V, et al. Familial isolated growth hormone deficiency is associated with increased systolic blood pressure, central obesity and dyslipidemia. Journal of Clinical Endocrinology and Metabolism 2002;87:2018-23.
11. Netchine I, Talon P, Dastot F, Vitaux F, Goosens M, Amselem S. Extensive phenotypic analysis of a family with growth hormone $(\mathrm{GH})$ deficiency caused by a mutation in the $\mathrm{GH}$-releasing hormone receptor gene. Journal of Clinical Endocrinology and Metabolism. 1998;83:432-6.

12. Salvatori R, Fan X, Phillips III JA, Espigares-Martin R, Martin De Lara I, Freeman $\mathrm{KL}$, et al. Three new mutations in the gene for the growth hormone $(\mathrm{GH})$-releasing hormone receptor in familial isolated GH deficiency type IB. Journal of Clinical Endocrinology and Metabolism. 2001;86:273-9.

13. Salvatori R, Fan X, Mullis PE, Haile A, Levine MA. Decreased expression of the growth hormone-releasing hormone receptor gene due to a mutation in a Pit-1 binding site. Mol Endocrinol. 2002;16:450-8

14. Salvatori R, Aguiar-Oliveira MH, Monte LBV, Hedges L, Santos NL, Pereira RMC, et al. Detection of a recurring mutation in the human growth hormone releasing hormone receptor gene. Clin Endocrinol (Oxf). 2002;57:77-80

15. Salvatori R, Fan X, Veldhuis J, Couch R. Serum GH response to pharmacological stimuli and physical exercise in two siblings with two new inactivating mutations in the $\mathrm{GH}$-releasing hormone receptor gene. Eur J Endocrinol. 2002;147:591-6.

16. Carakushansky M, Whatmore AJ, Clayton PE, Shalet SN, Gleeson HK, Price DA, et al. A new missense mutation in the growth hormone-releasing hormone receptor gene in familial isolated GH deficiency. Eur J Endocrinol. 2003;148:25-30.

17. Murray RA, Maheshwari HG, Russel EJ, Baumann G. Pituitary hypoplasia in patients with a mutation in the growth hormonereleasing hormone receptor gene. AJNR Am J Neuroradiol. 2000;21:685-9.

18. Osorio MG, Marui S, Jorge AA, Latronico AC, Lo LS, Leite CC, et al. Pituitary magnetic resonance imaging and function in patients with growth hormone deficiency with and without mutations in GHRH-R, GH-1, or PROP-1 genes. J Clin Endocrinol Metabol. 2002;87:5076-84.

19. Oliveira CRP, Salvatori R, Nóbrega LMA, Carvalho EOM, Menezes $\mathrm{M}$, Farias $\mathrm{CT}$, et al. Sizes of abdominal organs in adults with severe short stature due to severe, untreated, congenital $\mathrm{GH}$ deficiency caused by a homozygous mutation in the $\mathrm{GHRH}$ receptor gene. Clin Endocrinol (Oxf). 2007;22 Nov[Epub].

20. Barretto ESAB, Gill MS, Freitas MES, Magalhães MMG, Souza $\mathrm{AHO}$, Aguiar-Oliveira $\mathrm{MH}$, et al. Serum leptin and body composition in children with familial GH deficiency (GHD) due to a mutation in the growth hormone-releasing hormone (GHRH) receptor. Blackwell Science Ltd. 1999;51(5):559-64.

21. Gleeson HK, Souza AHO, Gill MS, Wieringa GE, Barretto ESA, Barretto-Filho JAS, et al. Lipid profiles in untreated severe congenital isolated growth hormone deficiency through the lifespan. Clin Endocrinol (Oxf). 2002;57:89-95.

22. Oliveira JL, Marques-Santos C, Barreto-Filho JA, Filho RX, Britto AV, Souza $A H$, et al. Lack of evidence of premature atherosclerosis in untreated severe isolated growth hormone deficiency due to a GHRH receptor mutation. Journal of Clinical Endocrinology and Metabolism. 2006;91:2093-9.

23. Gleeson HK, Barreto ESA, Salvatori R, Costa L, Oliveira CRP, Pereira RMC, et al. Metabolic effects of growth hormone (GH) replacement in children and adolescents with severe isolated $\mathrm{GH}$ deficiency due to a GHRH receptor mutation. Clin Endocrinol (Oxf). 2007;66:466-74.

24. Angelin B, Rudling M. Growth hormone and hepatic lipoprotein metabolism. Curr Opin Lipidol. 1994;5:160-5.

25. Parini $P$, Angelin B, Rudling M. Cholesterol and lipoprotein metabolism in aging: reversal of hypercholesterolemia by 
growth hormone treatment in old rats. Arterioscler Thromb Vasc Biol. 1999;19:832-9.

26. Hoogerbrugge $N$, Jansen $H$, Staels B, Kloet LT, Birkenhager JC. Growth hormone normalizes low-density lipoprotein receptor gene expression in hypothyroid rats. Metabolism. 1996;45:680-5.

27. Hew FL, Koschmann M, Christopher M, Rantzau C, Vaag A, Ward G, et al. Insulin resistance in growth hormone-deficient adults: defects in glucose utilization and glycogen synthase activity. J Clin Endocrinol Metab. 1996;81:555-64.

28. Gola M, Bonadonna S, Doga M, Guistina A. Clinical review. Growth hormone and cardiovascular risk factor. J Clin Endocrinol Metab. 2005;90:1864-70.

29. Bergman RN. Minimal model: perspective from 2005. Horm Res. 2005;64 Suppl 3:8-15.

30. Sneppen SP, Hoeck HC, Kollerup G, Sorensen OH, Laurberg P, Rasmussen UF. Bone mineral content and bone metabolism during physiological GH treatment in GH-deficient adults - an 18-month randomised, placebo-controlled, double blinded trial. Eur J Endocrinol. 2007;146:187-95.

31. Snyder PJ, Biller BM, Zagar A, Jackson I, Arah BM, Nippoldt $T B$, et al. Effect of growth hormone replacement on BMD in adult-onset growth hormone deficiency. J Bone Miner Res. 2007;22:762-70.

32. Paula FJA, Góis-Júnior MB, Aguiar-Oliveira MH, Pereira FA, Oliveira CRP, Pereira RMC, et al. Effects of short-term treatment with depot gh on bone in adults with isolated gh deficiency due to a mutation in the GHRH-receptor gene. Clin Endocrinol (Oxf). [In press].

33. Delafontaine P, Song YH, Li Y. Expression, regulation, and function of IGF-I, IGF-IR, and IGF-I binding proteins in blood vessels. Arterioscler Thromb Vasc Biol. 2004;24:435-44.
34. Oliveira JLM, Aguiar-Oliveira MH, D'Oliveira Jr. A, Pereira RMC, Oliveira CRP, Farias CT, et al. Congenital growth hormone $(\mathrm{GH})$ deficiency and atherosclerosis: effects of $\mathrm{GH}$ replacement in GH-naive adults. The Journal of Clinical Endocrinology \& Metabolism. 2007;92(12):4464-670.

35. Menezes M, Salvatori $R$, Oliveira CRP, Pereira RMC, Souza AHO, Nobrega LMA, et al. Climacteric in untreated isolated growth hormone deficiency. Menopause. 2007. [Epub].

36. Blum WF, Shavrikova EP, Edwards DJ, Rosilio M, Hartman ML, Marin F, et al. Decreased quality of life in adult patients with growth hormone deficiency compared with general populations using the new, validated, self-weighted questionnaire, questions on life satisfaction hipopituitarism module. J Clin Endocrinol Metab. 2003;88:4158-67.

37. Carroll PV, Christ ER, Bengtsson BA, et al. Growth hormone deficiency in adulthood and the effects of growth hormone replacement: a review. J Clin Endocrinol Metab. 1998;83:382-95.

38. Barbosa JAR. Qualidade de vida na deficiência isolada e genética do hormônio de crescimento $(\mathrm{GH})$. Efeitos da terapia de reposição com o GH. Dissertação. Sergipe: Universidade Federal de Sergipe, 2007.

\section{Endereço para correspondência:}

Manuel H. Aguiar-Oliveira

Departamento de Medicina, Universidade Federal de Sergipe

Rua Cláudio Batista, s/n

49060-100 Aracaju, SE

E-mail: herminio@infonet.com.br 